\title{
CLOSED OPERATORS COMMUTING WITH A WEIGHTED SHIFT ${ }^{1}$
}

ERIC A. NORDGREN

1. Introduction. A weighted shift operator with square summable weight sequence was used by Donoghue [2] to give the first example of an infinite unicellular lattice of invariant subspaces. It is our purpose here to prove two other striking properties of Donoghue operators (backwards shift operators on $l^{2}$ with positive, monotone, square summable weight sequence). First, if $S$ is a Donoghue operator, then the only closed operators that commute with $S$ are bounded, and second, these are all in the uniformly closed algebra generated by $S$. Although it is known that the commutant of $S$ is the weakly closed algebra generated by $S$, I believe that the result on uniform closure at least is new.

The commutativity property of the Donoghue operators should be contrasted with that of the backwards unilateral shift, which commutes with any unbounded coanaly tic Toeplitz operator.

2. Preliminaries. Our first result depends upon the structure of the invariant subspaces of $S \oplus S$ and upon a lemma connecting such subspaces with closed operators that commute with $S$. Recall that a closed operator is a linear transformation defined on a submanifold of a Hilbert space $\mathfrak{S}$ whose graph (as a subset of $\mathfrak{S} \oplus \mathfrak{F}$ ) is closed. A closed operator $A$ is said to commute with a bounded operator $T$ if and only if its domain is invariant under $T$ and $A T=T A$ on this domain.

Lemma 1. A closed operator commutes with a bounded operator $T$ if and only if its graph is an invariant subspace of $T \oplus T$.

I am grateful to Peter Rosenthal for calling this fact to my attention and suggesting its use in this investigation. It appeared at about the same time in his thesis and in a paper of Arveson [1, p. 639].

The invariant subspaces of $S \oplus S$ were described in [4], and we set forth here the relevant facts. The operator $S$ may be regarded as an operator on $H^{2}$, i.e. if $e_{n}(z)=z^{n}$ for $|z|=1, n=0,1,2, \cdots$, and $f=\sum \omega_{n} e_{n}$ is in $H^{2}$, then

$$
A f=\sum \alpha_{n} \omega_{n+1} e_{n},
$$

Received by the editors May 9, 1969.

1 Research supported in part by a grant from the National Science Foundation. 
where $\left\{\alpha_{n}\right\}$ is a monotonically decreasing square summable sequence of positive numbers. It will be convenient to regard elements of $H^{2} \oplus H^{2}$ either as ordered pairs $\langle f, g\rangle$ of functions, where $f$ and $g$ are in $H^{2}$, or as functions $\sum w_{n} e_{n}$, where $\left\{w_{n}\right\}$ is a norm square summable sequence in two-dimensional Hilbert space $C^{2}$. Then

$$
\langle f, g\rangle=\sum w_{n} e_{n}
$$

if and only if

$$
w_{n}=\left\langle\left(f, e_{n}\right),\left(g, e_{n}\right)\right\rangle
$$

for $n=0,1,2, \cdots$.

By Corollary 1 of Theorem 2 in [4], a nontrivial invariant subspace of $S \oplus S$ is the span $\mathfrak{M}\left(N,\left\{F_{n}\right\}\right)$ of the set of all "polynomials" $\left(\sum_{o}^{N-1} w_{n} e_{n}\right)$ of degree less than $N(N \geqq 0)$ and a finite or infinite sequence of vectors $F_{n}$ in $H^{2} \oplus H^{2}$. The vectors $F_{n}$ are defined in terms of an orthonormal basis $\{u, v\}$ for $C^{2}$ and a square summable sequence $\left\{\rho_{k}\right\}$ in $C$, the field of complex numbers, as follows:

$$
F_{0}=u e_{N}
$$

and if $n>0$, then

$$
F_{n}=u e_{N+n}+\sum_{j=0}^{n-1} \alpha(N, n, j) \rho_{n-j} v e_{N+j},
$$

where $\alpha(N, n, 0)=1$ and, if $j>0$,

$$
\alpha(N, n, j)=\left(\alpha_{N+n-1} \alpha_{N+n-2} \cdots \alpha_{N+n-j}\right) /\left(\alpha_{N} \alpha_{N+1} \cdots \alpha_{N+j-1}\right) .
$$

Finally, every vector in $\mathfrak{M}\left(N,\left\{F_{n}\right\}\right)$ may be represented as a sum $\sum_{o}^{N-1} w_{n} e_{n}+\sum_{o}^{\infty} \sigma_{n} F_{n}$, where $w_{n}$ is in $C^{2}$ for $n=0,1,2, \cdots, N-1$ and $\left\{\sigma_{n}\right\}$ is a square summable sequence in $C$.

Let $\mathfrak{M}\left(N,\left\{F_{n}\right\}\right)$ be an invariant subspace of $S \oplus S$, and let $u$ $=\langle\beta, \gamma\rangle$ and $v=\langle\delta, \epsilon\rangle$. Then we have the following lemma.

Lemma 2. Necessary and sufficient conditions that the only vector of the form $\langle 0, g\rangle$ in $\mathfrak{M}\left(N,\left\{F_{n}\right\}\right)$ be the zero vector are that $N=0$ and $\beta \neq 0$.

Proof. Necessity is immediate. Suppose $\mathfrak{M}\left(N,\left\{F_{n}\right\}\right)$ satisfies the conditions and $\langle 0, g\rangle$ is in $\mathfrak{M}\left(N,\left\{F_{n}\right\}\right)$. Then there is a square summable sequence $\left\{\sigma_{n}\right\}$ in $C$ such that

$$
\langle 0, g\rangle=\sum \sigma_{n} F_{n} .
$$

A simple calculation shows that 


$$
\begin{aligned}
\left(F_{n},\left\langle e_{k}, 0\right\rangle\right) & =0 \quad \text { if } k>n, \\
& =\beta \quad \text { if } k=n, \\
& =\delta \alpha(0, n, k) \rho_{n-k} \quad \text { if } k<n .
\end{aligned}
$$

If there were finitely many nonzero coefficients in (1), and $\sigma_{n}$ were the one with highest index, then (2) would imply that

$$
\langle 0, g\rangle=\left\langle\sigma_{n} \beta e_{n}+p, g\right\rangle,
$$

where $p$ is a polynomial of degree at most $n-1$. Since $\beta \neq 0$, this is impossible. Hence, either $\sigma_{n}=0$ for all $n$, or else $\sigma_{n} \neq 0$ for infinitely many $n$. Suppose the latter. Then on the one hand, (1) and (2) imply

$$
0=\left(\langle 0, g\rangle,\left\langle e_{k}, 0\right\rangle\right)=\sigma_{k} \beta+\delta \sum_{n=k+1}^{\infty} \alpha(0, n, k) \rho_{n-k} \sigma_{n} .
$$

But on the other hand, consider $k$ chosen so that

$$
\left|\sigma_{k}\right|>M_{k}=\max \left\{\left|\sigma_{n}\right|: n>k\right\},
$$

and use the Schwarz inequality to obtain

$$
\begin{aligned}
\mid \sigma_{k} \beta+\delta \sum_{n=k+1}^{\infty} \alpha & (0, n, k) \rho_{n-k} \sigma_{n} \mid \\
& \geqq\left|\sigma_{k} \beta\right|-|\delta| M_{k} \sum_{n=1}^{\infty} \alpha(0, n+k, k)\left|\rho_{n}\right| \\
& \geqq\left|\sigma_{k} \beta\right|-\delta M_{k}\left(\sum_{n=1}^{\infty}\left|\rho_{n}\right|^{2} \sum_{n=1}^{\infty} \alpha(0, n+k, k)^{2}\right)^{1 / 2} .
\end{aligned}
$$

Since $\alpha(0, n+k, k)<\alpha_{n+k-1} / \alpha_{o}$ and since $\left\{\alpha_{n}\right\}$ is square summable, the last term of (4) can be made positive by taking $k$ sufficiently large, in contradiction to (3). Hence $\langle 0, g\rangle$ is in $\mathfrak{M}\left(0,\left\{F_{n}\right\}\right)$ only if $g=0$.

CoRollary. The graph of a closed operator that commutes with $S$ is an invariant subspace $\mathfrak{M}\left(0,\left\{F_{n}\right\}\right)$ of $S \oplus S$ such that $\left(F_{o},\left\langle e_{o}, 0\right\rangle\right) \neq 0$.

3. Theorem. Every closed operator that commutes with a Donoghue operator is bounded.

Proof. Let $A$ be a closed operator that commutes with a Donoghue operator $S$. Then the graph of $A$ is an invariant subspace $\mathfrak{M}=\mathfrak{M}\left(0,\left\{F_{n}\right\}\right)$ of $S \oplus S$. We need only consider the case of infinitedimensional $\mathfrak{M}$. If $P_{1}$ (resp. $P_{2}$ ) is the projection of $\mathfrak{M}$ into $H^{2}$ that sends $\langle f, g\rangle$ into $f$ (resp. $g$ ), then $A=P_{2} P_{1}^{-1}$, and it will be enough to show that $P_{1}^{-1}$ is bounded. This will follow from the open mapping 
theorem if the range of $P_{1}$ can be shown to be closed. Since the sum of a finite-dimensional subspace and a (closed) subspace is automatically closed, it will suffice to show that $P_{1}$ is bounded away from zero on the span of $\left\{F_{n}: n>N\right\}$ for some $N$.

For each $n$, write $F_{n}=\left\langle f_{n}, g_{n}\right\rangle$ and $f_{n}=\beta e_{n}+\delta h_{n}$ where

$$
h_{n}=\sum_{j=0}^{n-1} \alpha(0, n, j) \rho_{n-j} e_{j} \text {. }
$$

Observe that the sequence $\left\{h_{n}\right\}$ is norm square summable in $H^{2}$. This follows from the inequality

$$
\begin{aligned}
\left\|h_{n}\right\|^{2} & =\left|\rho_{n}\right|^{2}+\left\|\sum_{j=1}^{n-1} \alpha(0, n, j) \rho_{n-j} e_{j}\right\| \\
& \leqq\left|\rho_{n}\right|^{2}+\left(\alpha_{n-1} / \alpha_{0}\right)^{2} \sum_{j=1}^{n-1}\left|\rho_{j}\right|^{2} .
\end{aligned}
$$

Choose $N$ large enough that

$$
\sum_{n=N}^{\infty}\left\|h_{n}\right\|^{2}<|\beta / 2|^{2}
$$

The projection of $\mathfrak{M}$ onto $H^{2} u$ is continuous, injective and epimorphic; therefore, it has a continuous inverse, of norm say $\mu$. Thus, if $\sum_{n=N}^{\infty} \sigma_{n} F_{n}$ is in $\mathfrak{M}$, then

$$
\begin{aligned}
\left\|P_{1} \sum_{n=N}^{\infty} \sigma_{n} F_{n}\right\| & =\left\|\beta \sum_{n=N}^{\infty} \sigma_{n} e_{n}+\delta \sum_{n=N}^{\infty} \sigma_{n} h_{n}\right\| \\
& \geqq|\beta|\left\|\sum_{n=N}^{\infty} \sigma_{n} e_{n}\right\|-\sum_{n=N}^{\infty}\left|\sigma_{n}\right|\left\|h_{n}\right\| \\
& \geqq|\beta / 2|\left\|\sum_{n=N}^{\infty} \sigma_{n} e_{n}\right\| \\
& \geqq|\beta / 2 \mu|\left\|\sum_{n=N}^{\infty} \sigma_{n} F_{n}\right\| .
\end{aligned}
$$

Hence $|\beta / 2 \mu|$ will serve as a lower bound, and the proof is complete.

4. Theorem. Every bounded operator that commutes with a Donoghue operator $S$ is in the uniform closure of the algebra generated by $S$.

Proof. Let $A$ be a bounded operator that commutes with $S$. By standard computations (cf. [3, p. 239]), $A$ has an upper triangular matrix $\left(a_{i j}\right)$, and if $\left\{a_{0}, a_{1}, a_{2}, \cdots\right\}$ is the sequence of entries in the 
uppermost row, then

$$
a_{i, i+k}=\alpha(0, i+k, i) a_{i}
$$

for $i>0$ and $k>0$. Another calculation shows that if

$$
p_{n}(z)=a_{o}+a_{1} z+\cdots+a_{n} z^{n},
$$

then $p_{n}(S)$ is an operator with a matrix whose diagonal and first $n$ superdiagonals coincide with those of the matrix of $A$. Thus, to complete the proof, it will be sufficient to show that

$$
\sum_{k=1}^{\infty} \sum_{i=0}^{\infty}\left|a_{i, i+k}\right|^{2}<\infty .
$$

But this is immediate from the inequality

$$
\left|a_{i, i+k}\right|<\left(\alpha_{k-1+i} / \alpha_{o}\right)\left|a_{k}\right|<\left(\alpha_{i} / \alpha_{o}\right)\left|a_{k}\right|,
$$

which follows from (1) and the monotonicity of the sequence $\left\{\alpha_{n}\right\}$, and from the fact (true for any bounded operator) that $\sum_{k=0}^{\infty}\left|a_{k}\right|^{2}<\infty$.

Corollary. Every closed operator that commutes with a Donoghue operator $S$ can be extended to a bounded operator in the uniform closure of the algebra generated by $S$.

\section{REFERENCES}

1. William B. Arveson, $A$ density theorem for operator algebras, Duke Math. J. 34 (1967), 635-647. MR $36 \# 4345$.

2. W. F. Donoghue, The lattice of invariant subspaces of a completely continuous, quasi-nilpotent transformation, Pacific J. Math 7 (1957), 1031-1035. MR 19, 1066.

3. P. R. Halmos, A Hilbert space problem book, Van Nostrand, Princeton, N. J., 1967. MR 34 \#8178.

4. E. A. Nordgren, Invariant subspaces of a direct sum of weighted shifts, Pacific J. Math. 27 (1968), 587-598.

UNIVERSITY OF NEW HAMPSHIRE 